\title{
Mid-infrared Thermo-optic Modulators in SOI
}

\author{
M. Nedeljkovic, S. Stanković, C. Mitchell, A. Z. Khokhar, S. Reynolds, D. J. \\ Thomson, F. Y. Gardes, C. G. Littlejohns, G. T. Reed, and G. Z. Mashanovich
}

\begin{abstract}
We report experimental results for thermo-optic modulators in silicon-on-insulator (SOI) material operating at the wavelength of $3.8 \mu \mathrm{m}$. These devices are based on asymmetric Mach-Zehnder interferometers (MZI) with aluminium heaters placed above one MZI arm. SOI rib waveguides with $400 \mathrm{~nm} \mathrm{Si}$ device layer thickness are used. Devices with conventional straight MZI arm and spiral MZI arm geometries are investigated. Straight-arm MZIs exhibited higher modulation depths, of up to $30.5 \mathrm{~dB}$, while spiral-arm MZIs required smaller switching powers, as low as $47 \mathrm{~mW}$. Measured -3 dB bandwidths were up to $23.8 \mathrm{kHz}$ and did not vary significantly with device configuration.
\end{abstract}

Index Terms-Thermo-optic modulator, silicon photonics, midinfrared, optoelectronic devices, photonics.

\section{INTRODUCTION}

$M$ ID-INFRARED (MIR) group IV photonics is attracting increasing attention for possible application in "lab-on-achip" technologies for chemical, biological and gas sensing as well as for free-space communications and infrared countermeasures [1]. Many of the passive components required to create an integrated mid-infrared photonic sensing system based in silicon-on-insulator (SOI) for wavelengths up to $4 \mu \mathrm{m}$ have now been demonstrated, such as waveguides [2-4], splitters/couplers [4], Mach-Zehnder interferometers [4], ring resonators [3] and spectrometers [5]. Planar electro-optical modulators have not yet been demonstrated in group-IV materials at wavelengths greater than $2.2 \mu \mathrm{m}$ [6], but are likely to have a part to play in the creation of high sensitivity sensing circuits. For experimental characterization of MIR components a combination of a chopper and lock-in amplifier is often used to increase the signal-to-noise ratio (SNR), e.g. [2, 7, 8], whose purpose is to shift the system to a higher operating frequency, where frequency dependent noise might be smaller [9].

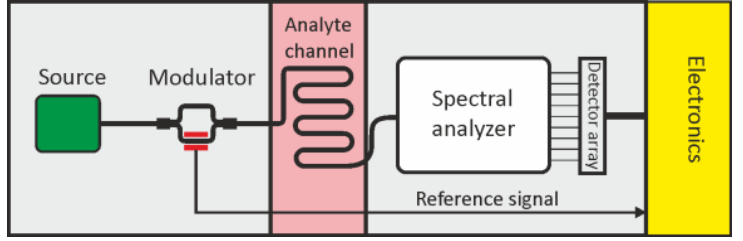

Fig. 1. Schematic diagram of integrated spectrometer, with a modulator acting as an on-chip chopper.

An electro-optical modulator could replace the chopper in an onchip equivalent of such a system, for example in the configuration shown in Fig. 1. In this paper we report characterization of thermooptic modulators operating at $3.8 \mu \mathrm{m}$.

The authors are with the Optoelectronics Research Centre, University of Southampton, Southampton, Hampshire, SO16 3HQ, United Kingdom. This work was supported by the UK Engineering and Physical Sciences Research Council (EPSRC) under the MIGRATION project and by the Royal Society

\section{THERMO-OPTIC MODULATOR DESIGN}

Thermo-optic modulators have been realised in near-IR (NIR) silicon components [10, 11], and although they exhibit much smaller modulation bandwidths than plasma dispersion effect modulators, they have been shown to be compact [11], require low powers $(6.5$ $\mathrm{mW}$ switching power [10]), and are much simpler to fabricate. In the NIR in SOI typical thermo-optic modulation bandwidths are in the 3.5$70 \mathrm{kHz}$ range [10], which is sufficient for sensing applications. In Fig. 2 the wavelength dependence of the thermo-optic coefficient, $d n / d T$, of silicon from [12] is shown. At $3.8 \mu \mathrm{m} d n / d T=1.70 \times 10^{-4}{ }^{\circ} \mathrm{C}^{-}$ ${ }^{1}$, which is $8 \%$ weaker than at $1.55 \mu \mathrm{m}$ but remains large enough for efficient thermo-optic modulation.

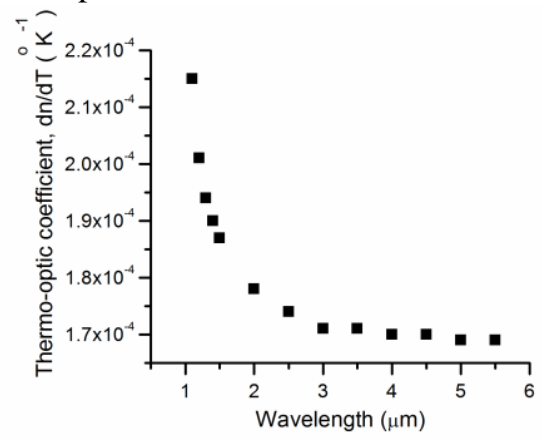

Fig. 2. Thermo-optic coefficient spectrum of silicon, plotted using the data of [12].

We investigate here modulators that are based on heaters placed above one arm of an asymmetric Mach-Zehnder interferometer (MZI). The MZI is based on SOI rib waveguides with silicon height $\mathrm{H}=400 \mathrm{~nm}$, waveguide width $\mathrm{W}=1300 \mathrm{~nm}$, etch depth $\mathrm{D}=220 \mathrm{~nm}$, and buried oxide layer $(\mathrm{BOX})$ thickness $\mathrm{H}_{\mathrm{BOX}}=2 \mu \mathrm{m} .1 \times 2$ multi-mode interferometers (MMIs) with tapered input and output ports are used as the splitter/coupler for the MZI. In [4] we showed that MMIs fabricated according to the same design and using the same fabrication process exhibited insertion losses of only $0.10 \pm 0.01 \mathrm{~dB}$. Waveguides with these dimensions, characterized using the cut-back method, showed a propagation loss of $3.5 \mathrm{~dB} / \mathrm{cm}$.

Densmore et al. [10] demonstrated thermo-optic modulators that employed both conventional MZIs with straight arms and MZIs with spiral patterned arms. The spiral-arm MZIs were found to require smaller switching powers (e.g. $6.5 \mathrm{~mW}$ for spiral-arm devices, and 36 $\mathrm{mW}$ for straight-arm devices), as when the waveguide is wrapped into a spiral the overlap between the heated volume and optical mode volume is improved. In this work we investigate devices based on both MZI types.

through Goran Mashanovich's Royal Society Research Fellowship. The corresponding author is Milos Nedeljkovic (e-mail: m.nedeljkovic@soton.ac.uk).

Copyright (c) 2014 IEEE. 
An aluminium heater, which acts as a phase shifter, is placed above one of the MZI arms (figures 3 and 4), and because there is high optical absorption in metals a $\mathrm{SiO}_{2}$ layer is used to isolate the heater from the optical mode. For the case of the straight-arm MZI the heater can be placed either directly above the waveguide, as in Fig. 3a, or with a slight lateral separation, as in Fig. 3b. The lateral heater separation further isolates the metal from the optical mode, but the increased distance from the waveguide will increase the required switching power, as a smaller proportion of the thermal power dissipated from the heater will heat the waveguide. For the spiral-arm case, the metal heating strip is folded such that it passes back and forth above the waveguide, as shown in Fig. $4 \mathrm{~b}$.
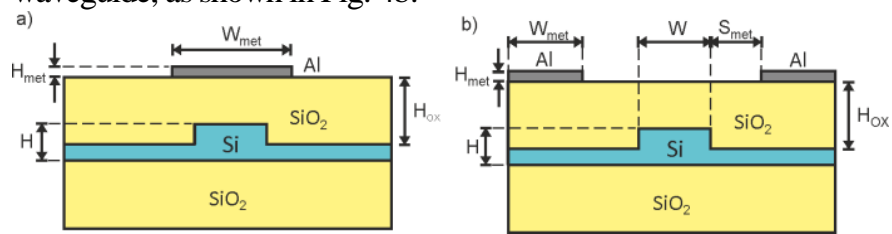

Fig. 3. Labelled schematic cross-section of thermo-optic phase shifters with metal heaters placed a) directly above the waveguide, b) with a slight lateral separation from the waveguide. Note that in practice the profile of deposited $\mathrm{SiO}_{2}$ is not flat, and is thicker above the waveguides.
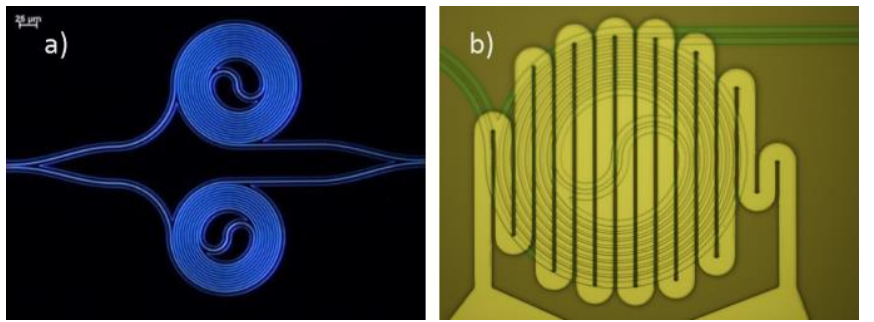

Fig. 4. Top view optical microscope image of a) a spiral-arm MZI, b) one arm of the spiral MZI with Al heater.

Fig. 5 shows the simulated waveguide loss with varying silica thickness when an aluminium strip is placed both directly above and separated laterally by $1 \mu \mathrm{m}$ from the waveguide. Simulations were carried out using the Photon Design Fimmwave software package, and the loss includes contributions from $\mathrm{Al}$ absorption $\left(5.6 \times 10^{6} \mathrm{~dB} / \mathrm{cm}\right.$ [13]) and $\mathrm{SiO}_{2}$ absorption (6 dB/cm [14]). It should be noted that in practice the absorption coefficient of the PECVD deposited $\mathrm{SiO}_{2}$ layer is likely to be different from the absorption of the BOX layer.

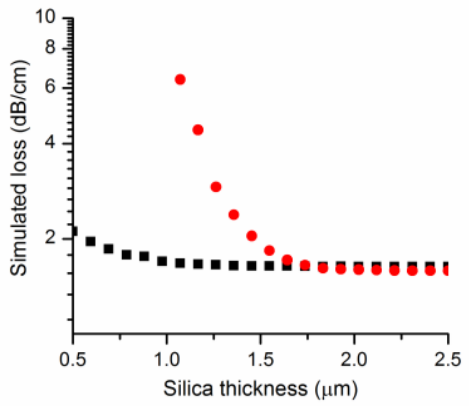

Fig. 5. Simulated optical absorption of waveguides with metal strips placed directly above waveguides (red dots) and metal strips with $1 \mu \mathrm{m}$ edge to edge separation from waveguides (black squares). In both cases the metal strip is 100 $\mathrm{nm}$ thick, and its width extends to the edge of the simulation region.

The figure shows that for negligible aluminium absorption loss $\mathrm{H}_{\mathrm{OX}}$ $>\sim 1 \mu \mathrm{m}$ is required for the laterally separated case, and $\mathrm{H}_{\mathrm{OX}}>\sim 1.8$ $\mu \mathrm{m}$ is required when the heater is directly above the waveguide. As both spiral-arm and straight-arm devices were fabricated on the same chip the silica isolating layer was chosen to be $2 \mu \mathrm{m}$ thick. As a conservative design choice the straight-arm modulators were chosen to have a separation $S_{\mathrm{met}}=1 \mu \mathrm{m}$ from the waveguide. Straight-arm devices were fabricated with heater widths of $10 \mu \mathrm{m}$ and $15 \mu \mathrm{m}$, and heater lengths of $2.25 \mathrm{~mm}$ to $4.5 \mathrm{~mm}$. The dimensions of spiral arm MZIs are defined by the spiral inner bend radius $R_{1}$, the edge to edge separation between waveguides in adjacent loops of the spiral $\mathrm{W}_{\text {gap }}$, and the number of spiral loops, N. Spiral-arm MZIs were fabricated with $R_{1}$ between $20 \mu \mathrm{m}$ to $42.5 \mu \mathrm{m}$ and $\mathrm{N}$ between 1 and 6 . From Fimmwave optical mode simulations it was determined that a separation $\mathrm{W}_{\text {gap }}=4 \mu \mathrm{m}$ is required so that the optical modes of waveguides in adjacent spiral loops do not interfere with each other. The resulting spirals had lengths between $0.7 \mathrm{~mm}$ and $9.1 \mathrm{~mm}$. The spiral-arm MZIs were made to be asymmetric by placing a spiral with $\mathrm{N}$ loops in one of the two arms, and $\mathrm{N}+1$ loops in the other.

\section{FABRICATION AND CHARACTERIZATION}

Chip fabrication was carried out at the Southampton Nanofabricaton Centre, University of Southampton. The waveguides were defined using e-beam lithography followed by ICP silicon etching. At this stage the wafer was diced into chips, and a $2 \mu \mathrm{m}$ thick layer of $\mathrm{SiO}_{2}$ was deposited on the chips by Plasma Enhanced Chemical Vapor Deposition (PECVD). A $120 \mathrm{~nm}$ thick Al layer was deposited on the chips by sputtering, and the aluminium heaters were patterned by standard contact photolithography followed by metal ICP etching.

\section{A. Optical characterization of Mach-Zehnder interferometers}

Optical characterization was carried out using a MIR experimental setup consisting of a quantum cascade laser operating at $3.8 \mu \mathrm{m}$ and an InSb detector with a time constant of $<1 \mu$ s [15], where light was coupled into and out of the chip using MIR fibers and grating couplers defined on the chip. The setup is described in much greater detail in [2]. A combination of a chopper in the optical beam operating at $\sim 250 \mathrm{~Hz}$ and a lock-in amplifier connected to the detector output were used to improve the SNR. Optical extinction ratios (ERs) and insertion losses for the fabricated devices were measured from wavelength scans in the range 3.725-3.80 $\mu \mathrm{m}$ that were normalized to the transmission through straight waveguides, for devices before $\mathrm{SiO}_{2}$ deposition. For straight-arm MZIs average extinction ratios across all of the devices were 19.3-22.5 dB, with the highest measured extinction ratio being $28.2 \mathrm{~dB}$, while average insertion losses were 1.3-2.2 dB. For spiral-arm MZIs maximum extinction ratios were 7.9-21.0 dB, while average insertion losses were 3.1-9.2 dB. The large variations in extinction ratio and insertion loss are mostly due to variations in spiral geometry. The greater insertion losses of spiral MZIs are attributed to bending loss in the spiral waveguides, particularly in the tight inner spiral layer. The insertion losses of both MZI types could be reduced by using waveguides with $\mathrm{W}=1350 \mathrm{~nm}$, which have been shown to have losses as low as $1.5 \mathrm{~dB} / \mathrm{cm}$ [4]. The smaller ERs of the spiral-arm MZI are partly attributed to a greater loss difference between the two arms than in the straight-arm MZI. Additionally, the free-spectral range (FSR) of the spiral-arm MZI is smaller, and as the QCL exhibits some wavelength instability due to mode-hopping behavior, it 
is more difficult to accurately interrogate these fine wavelength features.

\section{B. DC operation}

Electro-optical performance of the fabricated modulators was characterised under DC and AC operating conditions. An electrical probe was used to contact the heaters, which had probe pads at either end of the heating strip. For DC characterisation a PC controlled power supply was used to apply a steady current to the heater electrode. During the DC measurements the chopper was used, and scans of applied DC power versus optical power, measured from the lock-in amplifier output, were carried out. Fig. 6 shows an example of the measured normalized DC response of an asymmetric spiral-arm modulator with $\mathrm{R}_{1}=27.5 \mu \mathrm{m}$ and $\mathrm{N}=2$, with a spiral length of $1.77 \mathrm{~mm}$ and arm length difference of $96.1 \mu \mathrm{m}$. The red curve plotted alongside the measured points shows a fit of theoretical response of a $1 \times 2 \mathrm{MZI}$ [16]. Modulation depths (MD) have been calculated directly from the measured data, and switching power has been extracted from the fitted curve. Because $\mathrm{SiO}_{2}$ deposition was carried out on individual chips the $\mathrm{SiO}_{2}$ thickness was visibly uneven across sections of a sample, which meant that the transmission through MZIs could no longer be normalized to the transmission through straight waveguides for modulator insertion loss measurements.

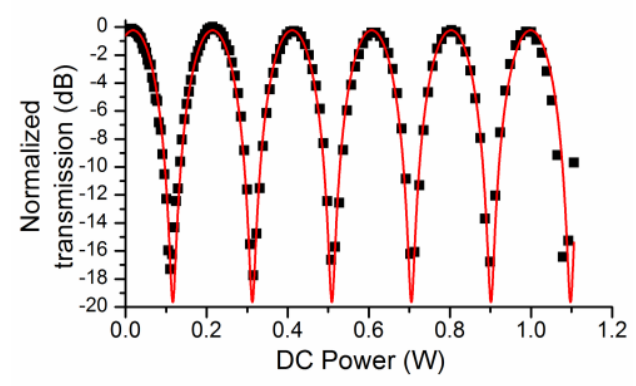

Fig. 6. Measured normalized transmission (black squares) of asymmetric spiral-arm MZI with $1.77 \mathrm{~mm}$ spiral length and $96.1 \mu \mathrm{m}$ arm length difference, plotted alongside a fitted theoretical curve (red line). $\mathrm{ER}=17.8 \mathrm{~dB}$.

The average DC modulation depth for straight-arm MZIs was 24.1 $\mathrm{dB}$ and the maximum MD was $30.5 \mathrm{~dB}$, while for spiral-arm MZIs the average $\mathrm{MD}$ was $10.9 \mathrm{~dB}$ and the maximum was $17.8 \mathrm{~dB}$, which was measured for the device shown in Fig. 6. The measured DC modulation depths are consistent with the optical extinction ratios measured from wavelength scans. Fig. 7a shows the power required to produce a $\pi$ phase shift plotted against heated waveguide length. It is clear that much greater switching powers are required for straight-arm MZIs than for spiral-arm MZIs, for which $47 \mathrm{~mW}$ is the smallest switching power, and that increasing the arm length of a spiral-arm MZI reduces the required switching power, but has little effect for straight-arm MZIs. This agrees with the findings of [10] for NIR modulators.
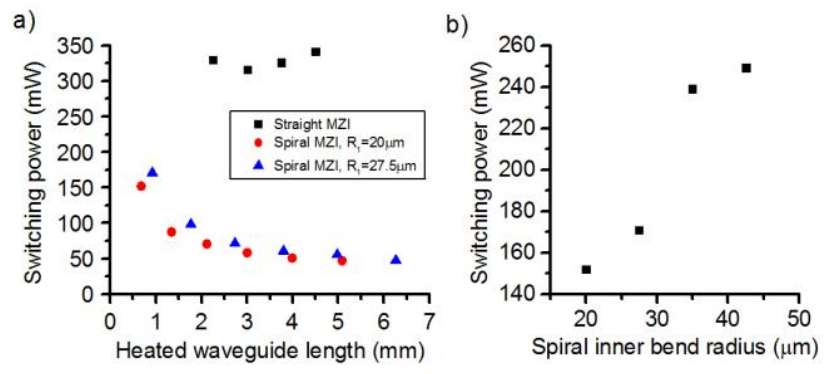

Fig. 7. a) Switching power of straight-arm MZIs (black squares) and of spiralarm MZIs with $R_{1}=20 \mu \mathrm{m}$ (red dots) and $R_{1}=27.5 \mu \mathrm{m}$ (blue triangles), plotted as a function of heated arm length. b) Switching power of spiral-arm MZIs with varying inner bend radius $\mathrm{R}_{0}$, but with the same waveguide width, waveguide separation and number of spiral loops.

The large switching powers of the straight-arm MZIs are largely a result of the lateral separation of the heater electrodes from the waveguide. This is confirmed by measurements on devices with the same waveguide edge to heater edge separation but different heater widths, and therefore different average separation. Devices with $\mathrm{W}_{\text {met }}$ $=10 \mu \mathrm{m}$ having heated arm lengths $\mathrm{L}_{\mathrm{H}}=2.25 \mathrm{~mm}$ and $3.0 \mathrm{~mm}$ required switching powers of $245 \mathrm{~mW}$ and $248 \mathrm{~mW}$ respectively, whereas those with $\mathrm{W}_{\text {met }}=15 \mu \mathrm{m}$ required $317 \mathrm{~mW}$ and $318 \mathrm{~mW}$ for those same heater lengths. For spiral-arm MZIs the switching power is also a function of spiral inner bend radius, as shown in Fig. 7b, which shows the switching power of spirals with the same $\mathrm{W}, \mathrm{W}_{\text {gap }}$, and $\mathrm{N}$, but different $R_{1}$. The switching power increases strongly with increasing inner bend radius despite an accompanying increase in heated spiral length, as there is an area at the centre of the spiral where the heater passes above etched silicon, therefore power used to heat this space is wasted. This is an important consequence of migrating spiral structures to longer wavelengths: longer wavelength spirals require greater bending radii and greater waveguide separation, limiting how tightly the spiral can be wrapped, and therefore limiting the efficiency of the modulator.

\section{AC operation}

For AC testing a PC controlled signal generator was used to apply a square wave signal to the heater electrode, and the mechanical chopper was removed from the optical path. The signal generator also supplied a reference signal to the lock-in amplifier, to filter the modulated frequency from the detector output. Fig. 8 shows the normalized optical transmission plotted against modulation frequency for a straight-arm MZI with $10 \mu \mathrm{m}$ wide heating strips and a $2.25 \mathrm{~mm}$ long heater. The $-3 \mathrm{~dB}$ bandwidth of the device shown in Fig. 8 was $23.8 \mathrm{kHz}$.

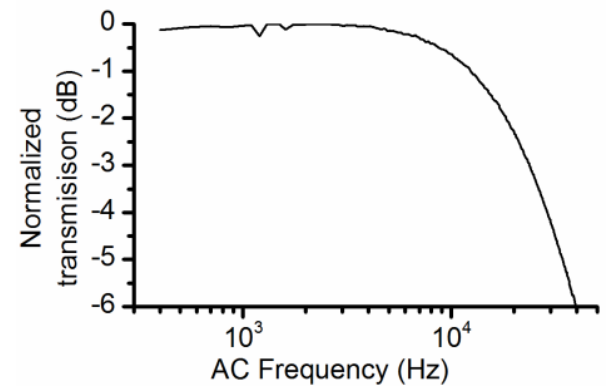

Fig. 8. Frequency response of a straight-arm MZI with $2.25 \mathrm{~mm}$ long heaters and $10 \mu \mathrm{m}$ wide heating strips. 
There was relatively little variation in the measured bandwidths of all of the measured devices regardless of layout, which were between $17.8 \mathrm{kHz}$ and $23.8 \mathrm{kHz}$, and no clear design dependent trends were discernible from the data. In [10] a $-3 \mathrm{~dB}$ bandwidth of $\sim 25 \mathrm{kHz}$ was measured for similar devices in the NIR, with the authors also noting that lateral heat diffusion has little effect on the frequency response, but that the switching speed could be increased by reducing the BOX thickness. However, at $3.8 \mu \mathrm{m}$ according to simulations a $2 \mu \mathrm{m}$ thick BOX layer results in substrate leakage of $0.6 \mathrm{~dB} / \mathrm{cm}$ for the waveguide dimensions used in this work, and further reduction of the BOX thickness would increase the propagation loss. A comparison of heater architectures in the literature [17] showed that there is a design tradeoff between reducing switching power and increasing modulation frequency, in which the speed of thermal dissipation is the determining factor.

\section{CONCLUSION}

In this paper we have demonstrated SOI thermo-optic modulators operating at $3.8 \mu \mathrm{m}$, in which an $\mathrm{Al}$ heater was placed above one arm of a Mach-Zehnder interferometer. MZIs with straight arms and MZIs with spiral arms were investigated. Straight-arm MZIs exhibited modulation depths of up to 30.5 $\mathrm{dB}$, insertion losses of 1.3-2.2 dB, $-3 \mathrm{~dB}$ bandwidths of up to $23.8 \mathrm{kHz}$, and power consumption of $245 \mathrm{~mW}$. Spiral-arm MZIs exhibited modulation depths of up to $17.8 \mathrm{~dB}$, insertion losses of 4.8-11.8 dB, depending on the configuration, $-3 \mathrm{~dB}$ bandwidths of up to $21.3 \mathrm{kHz}$, and power consumption of 47 $\mathrm{mW}$. The large power consumption of the straight-arm MZIs could be reduced by placing the $\mathrm{Al}$ heaters directly above the waveguide in the MZI arm, and by reducing the thickness of the $\mathrm{SiO}_{2}$ layer that separates the $\mathrm{Al}$ from the waveguide mode. The insertion losses of both MZI types could be reduced by using wider waveguides, which have previously been shown to have losses as low as $1.5 \mathrm{~dB} / \mathrm{cm}$ [4]. This would also be expected to increase the extinction ratio. The $-3 \mathrm{~dB}$ bandwidths are comparable to those that have been achieved in the literature for similar NIR devices. Ultimately, the power consumption of silicon MIR thermo-optic modulators will be greater than their NIR equivalents. The optical mode for longer wavelength waveguides is larger, therefore heaters must be placed further away from the waveguide, and in a spiral the waveguide folding density will be smaller because greater bend radii and greater separation between spiral layers are required. The thermo-optic effect is also weaker in the MIR than in the NIR. However, further optimization of the silica thickness and of the heater design for improved overlap between the heated area and the optical mode is required.

This device represents the first demonstration of a waveguide integrated silicon modulator at wavelengths greater than 2.2 $\mu \mathrm{m}$, and devices such as this could be important components of integrated mid-infrared sensing systems. As an on-chip chopper for sensing the thermo-optic modulator combines the ideal properties of low optical insertion loss and straightforward fabrication. For applications where faster modulation is needed modulators based on the free-carrier plasma dispersion effect should be investigated, and design equations for prediction of this effect in the MIR have recently become available [18].

\section{REFERENCES}

R. Soref, "Mid-infrared photonics in silicon and germanium," Nat Photon, vol. 4, pp. 495-497, 2010.

G. Z. Mashanovich, M. M. Milosevic, M. Nedeljkovic, N. Owens, B. Xiong, E. J. Teo, et al., "Low loss silicon waveguides for the midinfrared," Optics Express, vol. 19, pp. 7112-7119, 2011.

M. M. Milošević, M. Nedeljkovic, T. M. Ben Masaud, E. Jaberansary, H. M. H. Chong, N. G. Emerson, et al., "Silicon waveguides and devices for the mid-infrared," Applied Physics Letters, vol. 101, 121105, 2012.

M. Nedeljkovic, A. Z. Khokhar, Y. Hu, X. Chen, J. S. Penades, S. Stankovic, et al., "Silicon photonic devices and platforms for the mid-infrared," Optical Materials Express, vol. 3, pp. 1205-1214, 2013.

M. Muneeb, X. Chen, P. Verheyen, G. Lepage, S. Pathak, E. Ryckeboer, et al., "Demonstration of silicon-on-insulator midinfrared spectrometers operating at 3.8um," Optics Express, vol. 21, pp. 11659-11669, 2013.

M. A. Van Camp, S. Assefa, D. M. Gill, T. Barwicz, S. M. Shank, P. M. Rice, et al., "Demonstration of electrooptic modulation at $2165 \mathrm{~nm}$ using a silicon Mach-Zehnder interferometer," Optics Express, vol. 20, pp. 28009-28016, 2012.

T. Baehr-Jones, A. Spott, R. Ilic, A. Spott, B. Penkov, W. Asher, et al., "Silicon-on-sapphire integrated waveguides for the midinfrared," Optics Express, vol. 18, pp. 12127-12135, 2010.

A. Malik, M. Muneeb, S. Pathak, Y. Shimura, J. Van Campenhout, R. Loo, et al., "Germanium-on-silicon mid-infrared arrayed waveguide grating multiplexers," Photonics Technology Letters, IEEE, vol. 25, pp. 1805-1808, 2013.

R. Großkloß, P. Kersten, and W. Demtröder, "Sensitive amplitudeand phase-modulated absorption-spectroscopy with a continuously tunable diode laser," Applied Physics B, vol. 58, pp. 137-142, 1994. A. Densmore, S. Janz, R. Ma, J. H. Schmid, D.-X. Xu, A. Delâge, et al., "Compact and low power thermo-optic switch using folded silicon waveguides," Optics Express, vol. 17, pp. 10457-10465, 2009.

X. Wang, J. A. Martinez, M. S. Nawrocka, and R. R. Panepucci, "Compact thermally tunable silicon wavelength switch," in University/Government/Industry Micro/Nano Symposium, 2008. UGIM 2008. 17th Biennial, 2008, pp. 126-127.

B. J. L. Frey, Douglas B. ; Madison, Timothy J., "Temperaturedependent refractive index of silicon and germanium," in Proc. of SPIE, Orlando, USA, 2004.

E. D. Palik, Handbook of optical constants of solids vol. 1. San Diego: Academic Press, 1997.

R. A. Soref, S. J. Emelett, and W. R. Buchwald, "Silicon waveguided components for the long-wave infrared region," Journal of Optics A: Pure and Applied Optics, vol. 8, pp. 840-848, 2006.

E-mail correspondence with IR Associates Inc.

G. T. Reed, Silicon photonics: the state of the art: Wiley, 2008.

A. Masood, M. Pantouvaki, G. Lepage, P. Verheyen, J. Van Campenhout, P. Absil, et al., "Comparison of heater architectures for thermal control of silicon photonic circuits," in Group IV Photonics (GFP), 2013 IEEE 10th International Conference on, 2013, pp. 83-84.

M. Nedeljkovic, R. Soref, and G. Z. Mashanovich, "Free-carrier electrorefraction and electroabsorption modulation predictions for silicon over the 1.3-14um infrared wavelength range," Photonics Journal, IEEE, vol. 3, pp. 1171-1180, 2011. 